\title{
Analysis of Bacteriological Infected Dog and Cat Bite Wounds in Veterinary Medical Staff
}

\author{
Cristina RIMBU ${ }^{1}$, Cristina HORHOGEA ${ }^{1 *}$, Andreea COZMA ${ }^{1}$, Carmen CRETU ${ }^{1}$, Mariana GRECU ${ }^{1}$, Raluca \\ RUSU $^{1}$, Eleonora GUGUIANU ${ }^{1}$
}
${ }^{1}$ Department of Public Health, Faculty of Veterinary Medicine, The University of Agricultural Sciences and Veterinary Medicine Iaşi, Mihail Sadoveanu Alley 6-8, 700490 Iasi, Romania
*corresponding author: rebegeacristina@yahoo.com

Bulletin UASVM Veterinary Medicine 77(1)/2020

Print ISSN 1843-5270; Electronic ISSN 1843-5378

doi:10.15835/buasvmcn-vm: 2019.0038

\begin{abstract}
Bite wounds, human and animal origin, can lead to significant complications if appropriate therapy is not undertaken timeously. The major risk of these aggressions is given by the possibility of systemic propagation of the bacteria involved in the wound and the appearance of complications such as osteomyelitis, septic arthritis, bacterial endocarditis, etc. The identification of pathogenic germs and antibiotic sensitivity test were the key points for a specific therapy that could prevent the spread of infection in the deep tissues. Twenty five biological samples collected from various wounds produced by dogs and cats bites over the veterinary personnel during the medical evaluated. Various aerobic pathogenic and conditional pathogenic bacteria (Staphylococcus aureus, Staphylococcus pseudo intermedius, Staphylococcus intermedius, Staphylococcus epidermidis, Staphylococcus hominis, Staphylococcus xylosus, Staphylococcus warneri, Micrococcus roseus, Streptococcus sp. gr.B, Streptococcus sp. gr.F, Streptococcus sp. gr.G, Enterococcus faecalis, Enterococcus faecium, Trueperella pyogenes, Corynebacterium sp., Bacillus cereus, Pasteurella multocida, Pasteurella canis, Mannheimia haemolitica, Escherichia coli, Pseudomonas aeruginosa, Actinobacillus sp., Neisseria sp) and anaerobic bacteria (Clostridium perfringens, Bacteroides sp.) were isolated. The bacterial drug sensitivity was fluctuating, observing the variations influenced by the natural and acquired resistance of the tested bacterial species. The wounds produced by the bites of dogs and cats have aerobic and anaerobic polymicrobial etiology.
\end{abstract}

Keywords: bacteriologic analysis, infected bites

\section{Introduction}

Veterinary medical staff is frequently exposed to risk situations, including the aggression of the animals during examination. Even if the staff takes a proper approach to the animals and is trained to prevent such situations, however, the suffering animals become unpredictable and the rescuers become victims. The bites produced by these animals can cause injuries with varying degrees of severity, resulting in disabilities and local or systemic infections (Peters et al., 2004; Overall and Love, 2001).
Every wound is contaminated with microorganisms, but the infection is not always developed. However, during the bite produced by dogs and cats, the microorganisms are inoculated into the depths of the tissues and the risk of an infection is increased. The source of the infections is undoubtedly related to the bacterial microflora existing at the time of aggression in the oral cavity of the aggressor animals or the bacterial microflora present on the skin of the attacked person (Abrahamian and Goldstein, 2011). The etiology of these infections is most often polymicrobial, 
being isolated simultaneously from both aerobic (Pasteurella multocida, Staphylococcus aureus, Staphylococcus aureus, Staphylococcus intermedius, Actinomycesviscosus, etc. (Maraki et al., 2018; Blackburn et al., 2013) and anaerobic microorganisms (Porphyromonassp, Prevotella sp., Capnocytophaga canimorsus, Francisella tularensis, etc. (Blackburn et al., 2013; Brenner et al., 1989; Yaqub et al., 2004; Yuen and Malotky, 2011; Alexander et al. 1997). Mycoplasmas, chlamydia, viruses, etc. were not isolated from the bite wounds but there is a suspicion of their involvement, especially when the samples taken from the infected wounds are devoid of pathogenic microorganisms and the infection evolves (Abrahamian and Goldstein, 2011).

In Romania, there are few studies that have approached the subject from the perspective of quantifying bacterial biotypes in the wounds produced in humans by biting dogs and cats. Also, these wounds were superficially treated. Frequently, antibiotic therapy is applied without knowing the microbial aetiology and without performing an antibiogram. Under these conditions, the therapeutic failure occurs more and more often. The identification of pathogenic germs and antibiotic sensitivity test were were the key points for a specific therapy that could prevent the spread of infection in the deep tissues.

Therefore, we believe that this study may contribute to a better understanding of the infectious risk that appeared as a result of inoculation in a bitten wound, of a microbial population with a wide phenotypic, genotypic and pathogenic diversity.

\section{Materials and methods}

During 2017-2018, twenty five biological samples were harvested from wounds produced by biting of 18 dogs and 7 cats in veterinary medical personnel. All of these animals reacted during clinical examinations. The samples were collected using sterile swab under appropriate aseptic conditions. Sampling was carried out 3-24 hours after the time of aggression. The appearance of the lesions was different from one patient to another, depending on the intensity of the aggression.

The microbiological examination of these samples was performed at the Microbiology Laboratory of the Faculty of Veterinary Medicine of USAMV Iași.
The microbiological examination followed the classical diagnostic stages. The bacterioscopic examination of smears stained by Gram, Stamp and Methylene blue methods was followed by the isolation of aerobic and anaerobic bacteria using various culture media: Nutritional Broth, Nutrient Agar supplemented with 10\% defibrinated sheep blood for aerobes and VF Broth, Schneider horse blood agar, SPS (Sulfite Polymyxin Sulfadiazine Agar) for anaerobes. After 24-72 hours of incubation at $37^{\circ} \mathrm{C}$, under aerobic or anaerobic conditions, several types of bacterial colonies developed on the surface of culture media. Each type of colony has been transplanted on other special culture media: Chapmann (Oxoid), Levine (Oxoid), Bile esculineazide agar (Biorad). For selective isolation of pathogenic strains, chromogenic culture media were used: CHROMAgar MRSA, CHROMAgarStaph aureus, CHROMAgar VRE, CHROMAgar ESBL, GBS medium (Thermo Fisher Scientific). After another 24-48 hours of incubation, each colonial morphotype was evaluated by cultural, morphological and biochemical tests in order to identify the bacterial genus and species. For biochemical spectrum, complex identification systems API 20E, API 20NE, API 20A (Biomerieux), RapID тм STAPH PLUS System (Thermo Fisher Scientific) were used.

Bacterial strains sensitivity and resistance to antibiotics was performed by the diffusimetric method - disk diffusion test. For this purpose, a panel of 12 antibiotics most commonly used in wound therapy was selected: ampicillin $(10 \mu \mathrm{g})$, amoxicillin/clavulanic acid $(30 \mu \mathrm{g})$, gentamicin $(10 \mu \mathrm{g})$, clindamycin $(2 \mu \mathrm{g})$, enrofloxacin $(10 \mu \mathrm{g})$, marbofloxacin $(5 \mu \mathrm{g})$, ciprofloxacin $(5 \mu \mathrm{g})$, tetracycline $(30 \mu \mathrm{g})$, cephalexin $(30 \mu \mathrm{g})$, streptomycin $(25$ $\mu \mathrm{g})$, neomycin $(30 \mu \mathrm{g})$, fusidic acid $(10 \mu \mathrm{g})$.

\section{Results and discussions}

Following the microbiological exam, polymicrobial cultures were obtained, consisting of 33 (61.11\%) Gram positive strains and 21 (38.88\%) Gram negative strains (Table 1). This report is in favour of Gram positive bacteria and the explanation derives from the fact that Gram positive bacteria are commensal and quantitatively dominate human skin (Cosseau et al., 2016) and oral cavity mucosa in dogs and cats (Rîmbu, 2010), as well as in humans (Zawadzki et al., 2017). 
Table. 1. The frequency of bacterial strains isolated from bite wounds

\begin{tabular}{|c|c|c|c|c|}
\hline No. & & & BACTERIAL STRAINS & NO. STRAINS (\%) \\
\hline 1 & \multirow{17}{*}{ 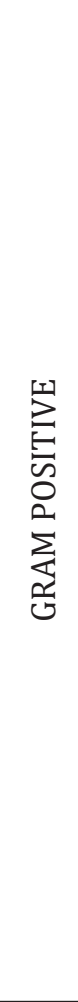 } & \multirow{16}{*}{ aerobic } & Staphylococcus aureus & $2(3.70 \%)$ \\
\hline 2 & & & Staphylococcus pseudointermedius & $5(9.26)$ \\
\hline 3 & & & Staphylococcus intermedius & $2(3.70)$ \\
\hline 4 & & & Staphylococcus epidermidis & $5(9.26)$ \\
\hline 5 & & & Staphylococcus hominis & $1(1.85)$ \\
\hline 6 & & & Staphylococcus xylosus & $1(1.85)$ \\
\hline 7 & & & Staphylococcus warneri & $1(1.85)$ \\
\hline 8 & & & Micrococcus roseus & $1(1.85)$ \\
\hline 9 & & & Streptococcus sp. gr.B & $1(1.85)$ \\
\hline 10 & & & Streptococcus sp. gr.F & $1(1.85)$ \\
\hline 11 & & & Streptococcus sp. gr.G & $1(1.85)$ \\
\hline 12 & & & Enterococcus faecalis & $2(3.70)$ \\
\hline 13 & & & Enterococcus faecium & $2(3.70)$ \\
\hline 14 & & & Trueperella pyogenes & $1(1.85)$ \\
\hline 15 & & & Corynebacterium sp. & $2(3.70)$ \\
\hline 16 & & & Bacillus cereus & $2(3.70)$ \\
\hline 17 & & anaerobic & Clostridium perfringens & $3(5.55)$ \\
\hline & \multirow{9}{*}{ 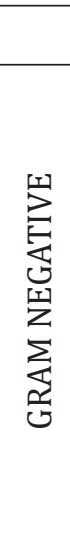 } & & TOTAL GRAM POSITIVE & $33(61.12)$ \\
\hline 18 & & \multirow{7}{*}{ aerobic } & Pasteurella multocida & $6(11.11)$ \\
\hline 19 & & & Pasteurella canis & $3(5.55)$ \\
\hline 20 & & & Mannheimia haemolitica & $1(1.85)$ \\
\hline 21 & & & Escherichia coli & $6(11.11)$ \\
\hline 22 & & & Pseudomonas aeruginosa & $2(3.70)$ \\
\hline 23 & & & Actinobacillus sp. & $1(1.85)$ \\
\hline 24 & & & Neisseria sp. & $1(1.85)$ \\
\hline \multirow[t]{3}{*}{25} & & anaerobic & Bacteroides sp. & $1(1.85)$ \\
\hline & & & TOTAL GRAM NEGATIVE & $21(38.88)$ \\
\hline & \multicolumn{3}{|c|}{ TOTAL BACTERIAL STRAINS } & $54(100)$ \\
\hline
\end{tabular}

Four $(7.4 \%)$ of the $54(100 \%)$ isolated strains were anaerobic: Clostridium perfringens and Bacteroides $s p$.) and 50 (92.6\%) were aerobic or facultative anaerobic strains: Staphylococcus aureus, Staphylococcus pseudointermedius, Staphylococcus intermedius, Staphylococcus epidermidis, Staphylococcus hominis, Staphylococcus xylosus, Staphylococcus warneri, Micrococcus roseus, Streptococcus sp. gr.B, Streptococcus sp. gr.F, Streptococcus sp. gr.G, Enterococcus faecalis, Enterococcus faecium,
Trueperella pyogenes, Corynebacterium sp., Bacillus cereus, Pasteurella multocida, Pasteurella canis, Mannheimia haemolitica, Escherichia coli, Pseudomonas aeruginosa, Actinobacillus sp., Neisseria $s p$ (Figure 1).

Most bacterial species isolated from the bite wounds are part of the oral microflora of dogs and cats (Rîmbu, 2010; Abrahamian and Goldstein, 2011). Some bacterial strains considered to be specific for human skin have been isolated 


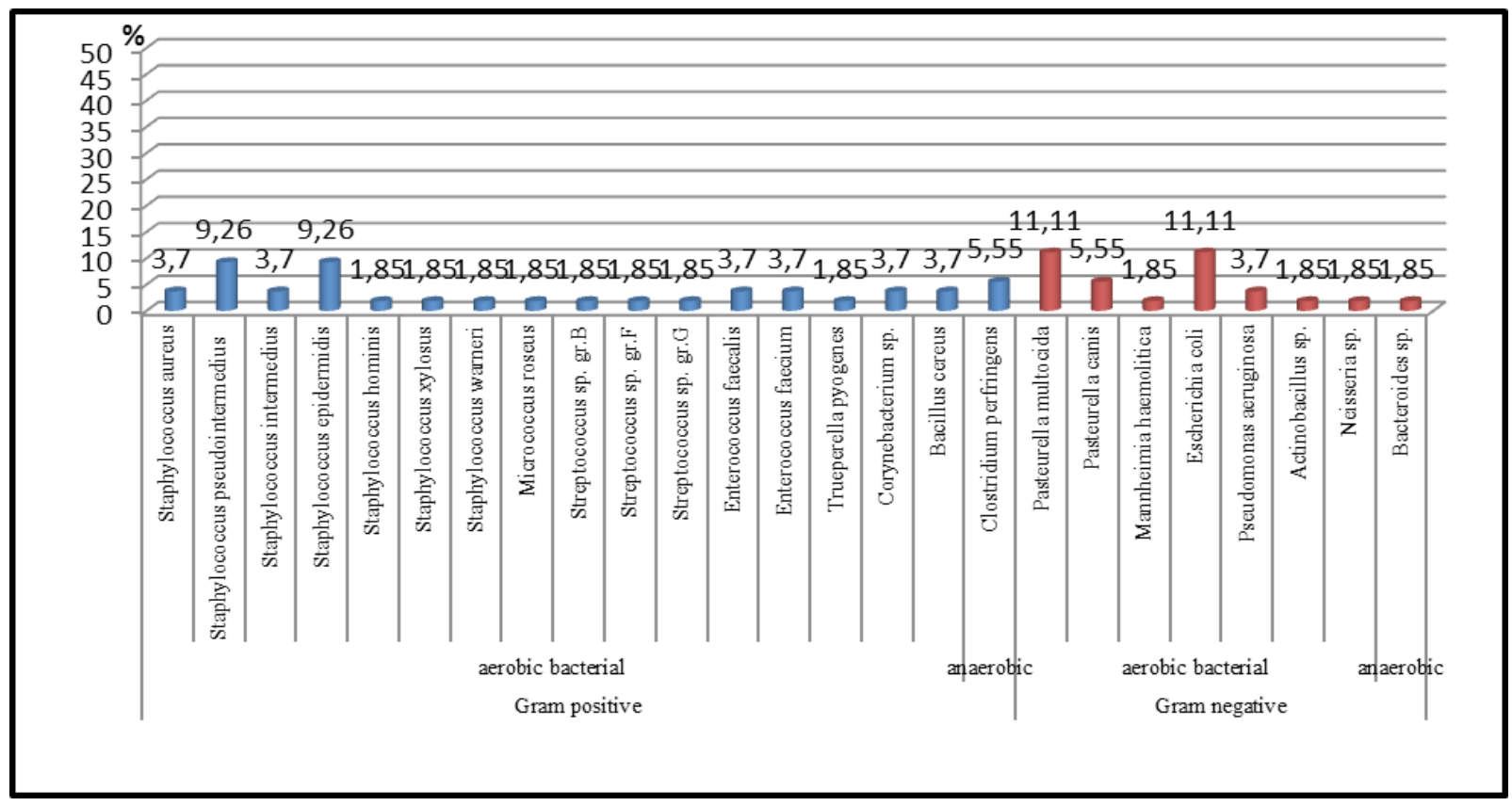

Figure 1. Graphical representation of the frequency of isolation of bacterial strains from the bites wound

from the same samples: Staphylococcus aureus, Staphylococcus epidermidis, Staphylococcus hominis, although there are studies showing that these species can transit the mucosa of the pet's oral cavity as a result of common cohabitation (Rîmbu, 2010; Abrahamian and Goldstein, 2011, Rîmbu et al. 2012 ).

This study highlights the dominant involvement of staphylococci and species of the genus Pasteurella and Escherichia coli. Staphylococcus aureus is commonly involved in producing dermatitis, septicaemia and food poisoning in humans. Staphylococcus intermedius and Staphylococcus pseudointermedius are considered the most important non-aureus species, identified in the skin of pets (Gyles et al., 2004). Pasteurella multocida and Pasteurella canis are the most common species isolated from human infected bite wounds (Abrahamian and Goldstein, 2011). These bacterial species and Pasteurella multocida, in particular, have a very good systemic propagation capacity, being responsible for the appearance of abscesses, lymphatic infections (Rao and Jain, 1999) and the toxins they release in the body affect the central nervous system (Nielsen et al, 1986). Escherichia coli appear in bacterial communities with low involvement in biting wound infections (Abrahamian and Goldstein, 2011). However, it was frequently isolated from the samples analyzed in our study, which shows that, most likely, no adequate care measures were taken after the trauma occurred.

Although Enterococcus faecalis (3.7\%) and Enterococcus faecium (3.7\%) have been isolated with a low frequency and are classified with low pathogenicity, the importance of these species as triggers of nosocomial infections and infectious syndromes is related to the natural and acquired resistance to some antibiotics (Maki and Agger, 1988). Given that their isolation was accomplished in mixed cultures and the antibiogram was performed on the total microflora, in many cases the therapeutic options are reduced.

With a much lower frequency (1.85\%), groups $\mathrm{B}, \mathrm{F}$ and $\mathrm{G}$ streptococci were isolated, and their presence, especially groups $B$ and $G$, is responsible for the appearance of the necrotizing fasciitis (DiNubile and Lipsky, 2004). Group G of streptococci includes the zoonotic species Streptococcus canis, responsible for the development of infectious endocarditis and septicaemia in humans (Galperine et al., 2007).

Trueperella pyogenes $(1.85 \%)$ and non identified strains of the genus Corynebacterium (3.7\%), Actinobacillus (1.85\%) and Neiseria (3.7\%) belong to the common bacterial communities, frequently isolated from the bite wounds produced by animals in humans (Talan et al., 1999). 
Escherichia coli and Pseudomonas aeruginosa are part of the normal intestinal microbiota of humans and animals and have a special role in nosocomial epidemiology given the ease with which they acquire multiple resistances to antibiotics (Carp Carare et al., 2015; Guentzel, 1996). Isolation of these opportunistic species from the bite wounds reflects their ecological variability and their pathogenic potential.

Anaerobic bacteria play an important role in infecting the deep wounds produced by animal bites, especially at the upper extremities of humans (Talan et al., 1999). Isolation of these types of bacteria is difficult, which is probably why only two bacterial types have been isolated: Clostridium perfringens (5.55\%) and Bacteroides $s p .(1.85 \%)$. The absence of anaerobes in the samples does not exclude their presence in the plagues (Abrahamian and Goldstein, 2011).

All these pathogenic or conditioned pathogenic species are opportunistic, and their isolation from the samples taken from the infected wounds produced by the bites of dogs and cats, prove their ability to adapt and the possibility of their involvement in triggering the infectious process.

Most bacterial strains have been tested to determine the sensitivity profile to antibiotics. The table 2 summarizes the results of the antimicrobial susceptibility testing. Analyzing the sensitivity profile, it is found that isolated bacterial biotypes respond to most antibiotics, but in different ways.

Table 2. Numerical distribution of bacterial strains relative to antibiotic sensitivity profile

\begin{tabular}{|c|c|c|c|c|c|c|c|c|c|c|c|c|c|c|}
\hline Bacterial species & 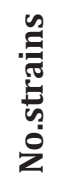 & & $\sum_{<}$ & $\sum_{\varepsilon}^{U}$ & Z & $\overleftarrow{a}$ & 电 & $\sum_{\Sigma}^{\mathscr{c}}$ & $\hat{v}$ & 受 & $\stackrel{0}{\oplus}$ & us & ت & 오 \\
\hline \multirow{3}{*}{ Staphylococcus sp. } & \multirow{3}{*}{14} & $\mathrm{~S}$ & 5 & 7 & 9 & 13 & 9 & 11 & 12 & 10 & 7 & 8 & 9 & 9 \\
\hline & & MS & 9 & 6 & 5 & 1 & 4 & 3 & 3 & 4 & 7 & 6 & 4 & 5 \\
\hline & & $\mathrm{R}$ & 0 & 1 & 0 & 0 & 1 & 0 & 0 & 0 & 0 & 0 & 1 & 0 \\
\hline \multirow{3}{*}{ Streptococcus sp. } & \multirow{3}{*}{3} & $\mathrm{~S}$ & 3 & 3 & 2 & 0 & 0 & 2 & 3 & 2 & 0 & 0 & 2 & 2 \\
\hline & & MS & 0 & 0 & 1 & 3 & 3 & 1 & 0 & 1 & 3 & 3 & 1 & 1 \\
\hline & & $\mathrm{R}$ & 0 & 0 & 0 & 0 & 0 & 0 & 0 & 0 & 0 & 0 & 0 & 0 \\
\hline \multirow{3}{*}{ Enterococcus sp. } & \multirow{3}{*}{4} & $\mathrm{~S}$ & 1 & 1 & 0 & 2 & 2 & 2 & 3 & 1 & 0 & 0 & 1 & 0 \\
\hline & & MS & 3 & 3 & 4 & 1 & 2 & 2 & 1 & 3 & 4 & 4 & 3 & 3 \\
\hline & & $\mathrm{R}$ & 0 & 0 & 0 & 1 & 0 & 0 & 0 & 0 & 0 & 0 & 0 & 1 \\
\hline \multirow{3}{*}{$\begin{array}{c}\text { Trueperella sp./ } \\
\text { Corynebacterium } \\
\text { sp. }\end{array}$} & \multirow{3}{*}{3} & $\mathrm{~S}$ & 0 & 3 & 0 & 3 & 3 & 3 & 3 & 3 & 0 & 2 & 2 & 0 \\
\hline & & MS & 3 & 0 & 3 & 0 & 0 & 0 & 0 & 0 & 3 & 0 & 1 & 2 \\
\hline & & $\mathrm{R}$ & 0 & 0 & 0 & 0 & 0 & 0 & 0 & 0 & 0 & 1 & 0 & 1 \\
\hline \multirow{3}{*}{ Clostridium sp. } & \multirow{3}{*}{3} & $\mathrm{~S}$ & 2 & 2 & 0 & 0 & 1 & 1 & 2 & 2 & 0 & 1 & 1 & 0 \\
\hline & & MS & 1 & 1 & 2 & 3 & 2 & 2 & 1 & 1 & 3 & 2 & 2 & 2 \\
\hline & & $\mathrm{R}$ & 0 & 0 & 1 & 0 & 0 & 0 & 0 & 0 & 0 & 0 & 0 & 1 \\
\hline \multirow{3}{*}{$\begin{array}{l}\text { Pasteurella sp / } \\
\text { Mannheimia sp. }\end{array}$} & \multirow{3}{*}{10} & $\mathrm{~S}$ & 5 & 9 & 7 & 3 & 6 & 6 & 9 & 7 & 6 & 2 & 6 & 7 \\
\hline & & MS & 4 & 1 & 2 & 6 & 4 & 4 & 1 & 3 & 2 & 8 & 4 & 2 \\
\hline & & $\mathrm{R}$ & 1 & 0 & 1 & 1 & 0 & 0 & 0 & 0 & 2 & 0 & 0 & 1 \\
\hline \multirow{3}{*}{ Escherichia sp. } & \multirow{3}{*}{6} & $\mathrm{~S}$ & 4 & 3 & 3 & 2 & 2 & 3 & 4 & 3 & 4 & 4 & 4 & 4 \\
\hline & & MS & 2 & 1 & 3 & 4 & 2 & 3 & 1 & 3 & 2 & 2 & 2 & 2 \\
\hline & & $\mathrm{R}$ & 0 & 2 & 0 & 0 & 2 & 0 & 0 & 0 & 0 & 0 & 0 & 0 \\
\hline \multirow{3}{*}{ Pseudomonas sp. } & \multirow{3}{*}{2} & $\mathrm{~S}$ & 0 & 0 & 0 & 0 & 0 & 1 & 1 & 0 & 1 & 0 & 0 & 0 \\
\hline & & MS & 1 & 2 & 2 & 1 & 2 & 1 & 1 & 2 & 1 & 2 & 1 & 2 \\
\hline & & $\mathrm{R}$ & 1 & 0 & 0 & 1 & 0 & 0 & 0 & 0 & 0 & 0 & 1 & 0 \\
\hline \multirow{3}{*}{ Actinobacillus sp. } & \multirow{3}{*}{1} & $\mathrm{~S}$ & 1 & 1 & 0 & 1 & 1 & 1 & 1 & 0 & 0 & 0 & 1 & 0 \\
\hline & & MS & 0 & 0 & 1 & 0 & 0 & 0 & 0 & 1 & 1 & 1 & 0 & 1 \\
\hline & & $\mathrm{R}$ & 0 & 0 & 0 & 0 & 0 & 0 & 0 & 0 & 0 & 0 & 0 & 0 \\
\hline \multirow{3}{*}{ Bacteroides sp. } & \multirow{3}{*}{1} & $\mathrm{~S}$ & 0 & 0 & 0 & 1 & 0 & 0 & 1 & 0 & 0 & 0 & 0 & 0 \\
\hline & & MS & 1 & 1 & 0 & 0 & 1 & 1 & 0 & 0 & 1 & 1 & 1 & 0 \\
\hline & & $\mathrm{R}$ & 0 & 0 & 1 & 0 & 0 & 0 & 0 & 1 & 0 & 0 & 0 & 1 \\
\hline
\end{tabular}

Note:S=Susceptibile; $M S=$ Moderate Sensitivity $; R=$ Resistant

$\mathrm{AM}=$ ampicillin, $\mathrm{AMC}=$ amoxicillin/clavulanic acid; $\mathrm{GN}=$ gentamycin, $\mathrm{DA}=$ clindamycin, $\mathrm{ENR}=$ enrofloxacina, $\mathrm{MAR}=$ marbofloxacin, $\mathrm{CIP}=$ ciprofloxacin, $\mathrm{Ne}=$ neomycin, $\mathrm{Te}=$ Tetracycline, $\mathrm{S}=$ streptomicyn, $\mathrm{CL}=$ cefalexin, $\mathrm{FD}=$ fusid acid 
Testing the 14 Staphylococcus sp. strains with 12 antimicrobial substances panel revealed a good sensitivity that offered many therapeutic possibilities (Figure 2).

The efficiency of antimicrobial substances was evaluated according to the number of strains that were inhibited by their action. Thus, the most effective antibiotics against staphylococci were: clindamycin $(n=13)$, ciprofloxacin $(n=12)$, marbofloxacin $(\mathrm{n}=11)$, neomycin $(\mathrm{n}=10)$, enrofloxacin $(n=9)$, gentamicin $(n=9)$, cephalexin $(n=9)$, streptomycin ( $\mathrm{n}=9$ ).

The same panel of antibiotics was tested on the 3 strains of Streptococcus $s p$. and the results showed a very good sensitivity to ampicillin $(n=3)$, amoxicillin-clavulanic acid ( $\mathrm{n}=3)$ and ciprofloxacin $(n=3)$ (Figure 3). All streptococcus strains had moderate sensitivity to streptomycin, clindamycin, tetracycline and enrofloxacin, antibiotics that are commonly used in the therapy of infections produced by these microorganisms.

The 4 strains of Enterococcus sp. were predominantly moderately sensitive to antibiotics: gentamicin $(n=4)$, tetracycline $(n=4)$, streptomycin ( $n=4)$, ampicillin $(n=3)$, amoxicillin clavulanic acid $(n=3)$, cephalexin $(n=3)$ and fusidic acid $(n=3)$ (Figure 4).

Only one strain of Trueperella sp. and 2 strains of Corynebacterium $s p$ were isolated from the wounds of the patients, which were sensitive to most of the antibiotics tested: amoxicillin + clavulanic acid, clindamycin, enrofloxacin, marbofloxacin, ciprofloxacin and neomycin but which had streptomycin and fusidic acid (Figure 5).

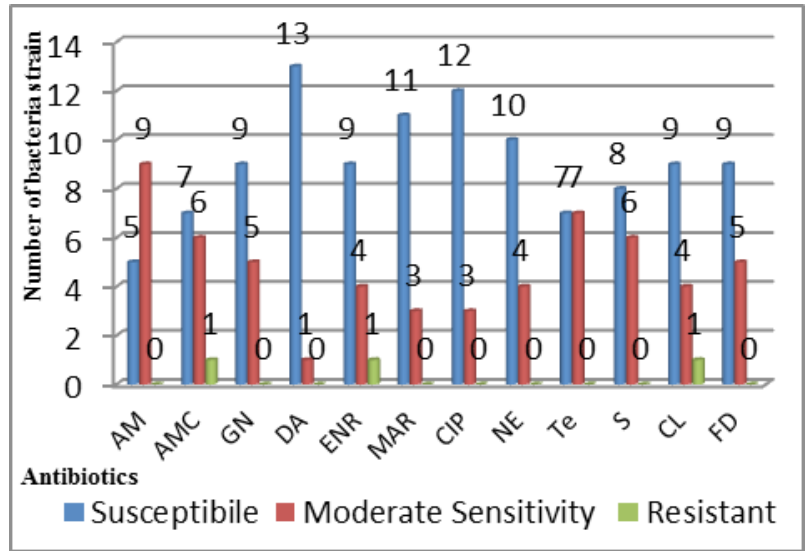

Figure 2. Antibiotic susceptibility profile of Staphylococcus sp.

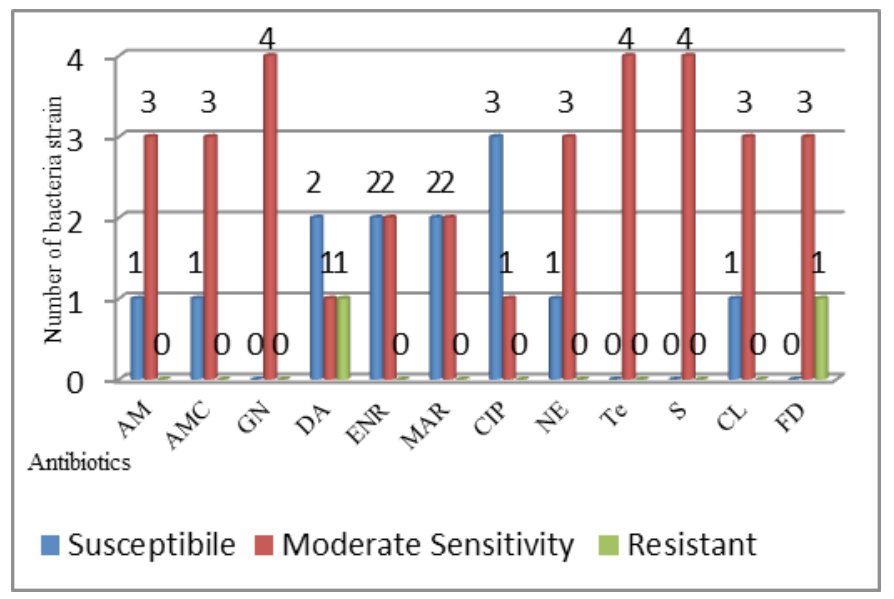

Figure 4. Antibiotic susceptibility profile of Enterococcus sp.

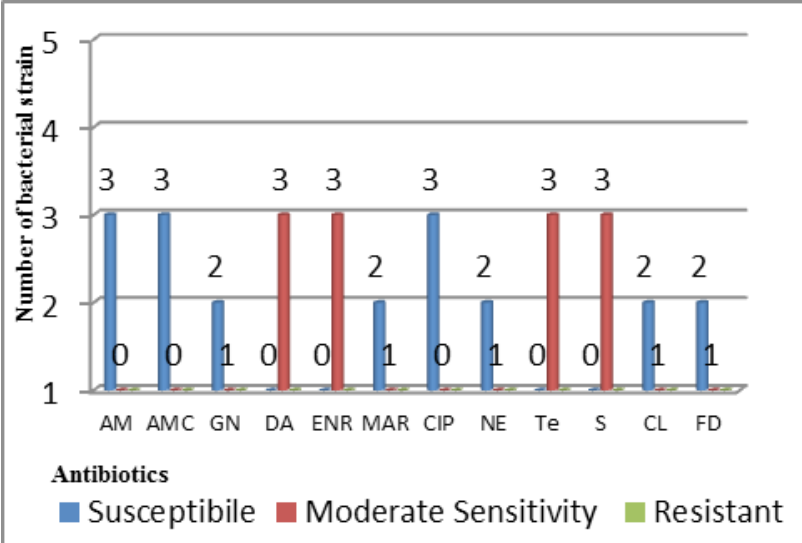

Figure 3. Antibiotic susceptibility profile of Streptococcus sp.

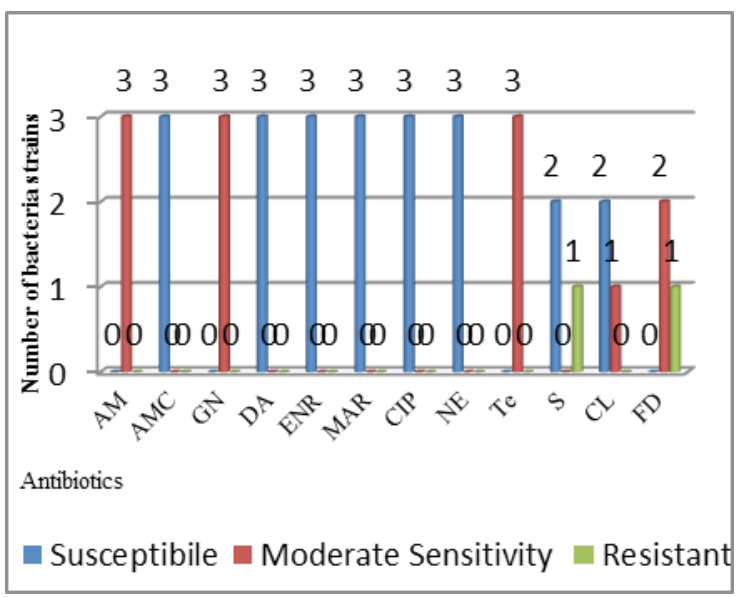

Figure 5. Antibiotic susceptibility profile of Trueperella sp./Corynebacterium sp. 


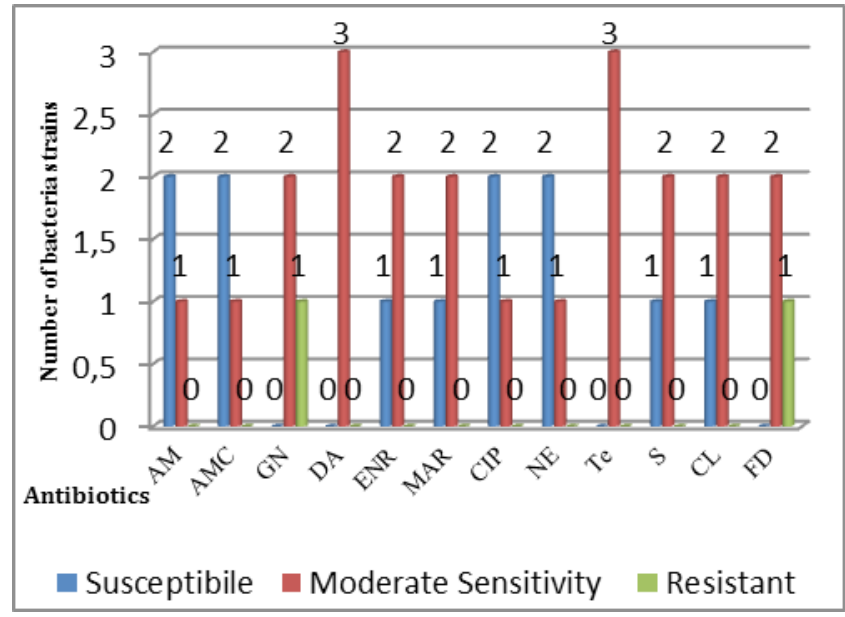

Figure 6. Antibiotic susceptibility profile of Clostridium sp.

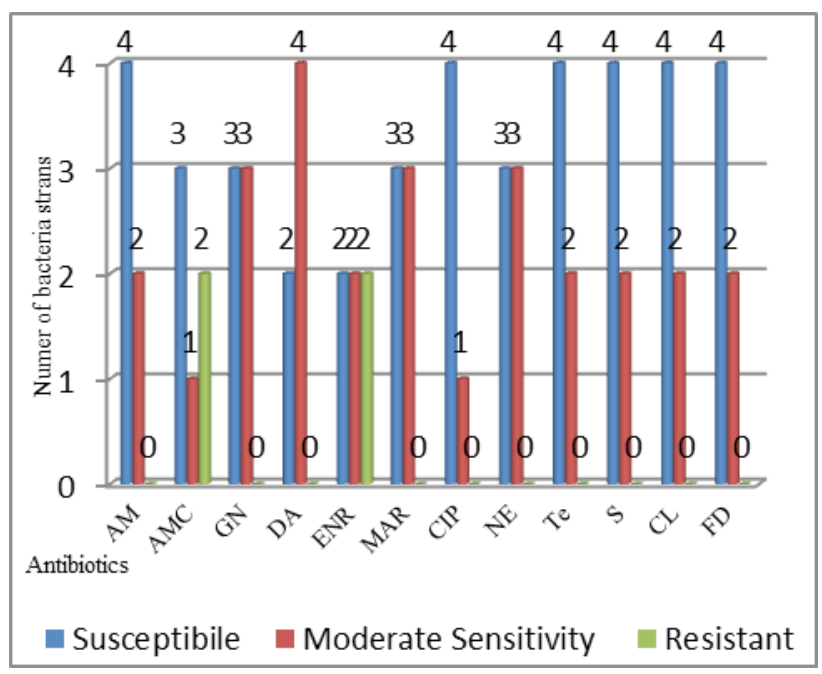

Figure 8. Antibiotic susceptibility profile of Escherichia sp.

The 3 Clostridium sp. strains isolated from the pathological samples were tested against the same panel of antibiotics used in human wound therapy. The most active antimicrobial agents against these Gram-positive anaerobic strains were: ampicillin, amoxicillin + clavulanic acid, ciprofloxacin and neomycin (Figure 6).

Of the Gram-negative bacteria, the most isolated species belonged to the genus Pasteurella / Mannheimia $(\mathrm{n}=10)$, which showed very good sensitivity to the following antibiotics: amoxicillin + clavulanic acid ( $n=9)$, ciprofloxacin $(n=9)$, gentamicin ( $n=7)$, neomycin ( $n=7)$, fusidic acid $(n=7)$ (Figure 7).

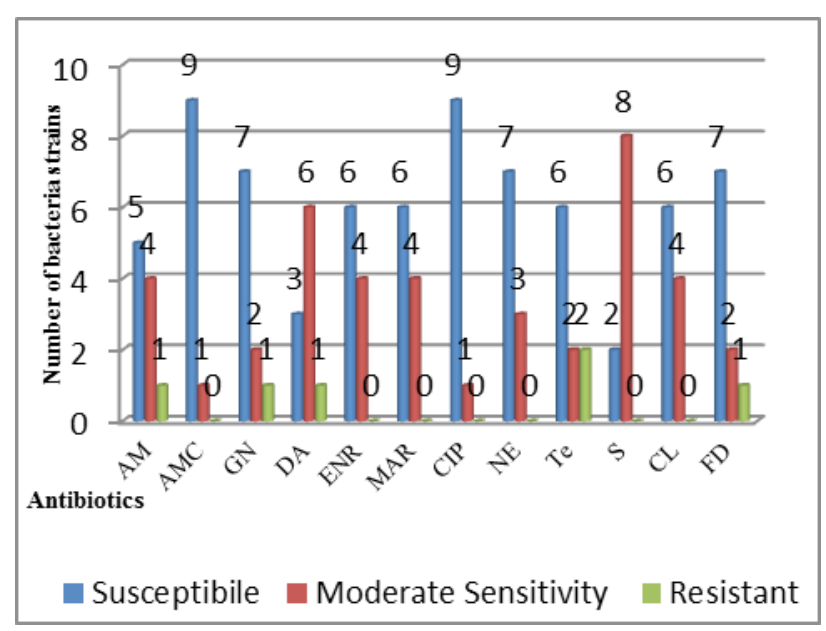

Figure 7. Antibiotic susceptibility profile of Pasteurella sp./Manheimia sp.

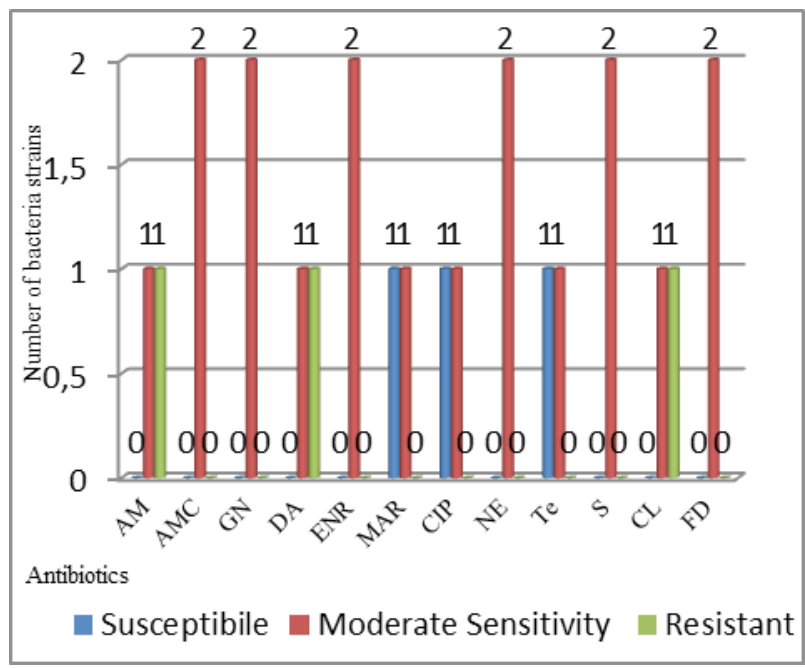

Figure 9. Antibiotic susceptibility profile of Pseudomonas sp.

The presence of enterobacteria together with the other pathogenic Gram-negative bacterial species (Pasteurella, Manheimia, Pseudomonas), shows the high degree of fecaloid contamination of the wounds produced by the bites of the pets. After testing the sensitivity to the selected antibiotics, it was revealed the fact that the most active antibiotics against Escherichia sp. strains were: ampicillin $(\mathrm{n}=4)$, ciprofloxacin $(\mathrm{n}=4)$, tetracycline $(n=4)$, streptomycin $(n=4)$, cephalexin $(n=4)$ and fusidic acid $(\mathrm{n}=4)$ (Figure 8).

The 2 isolated Pseudomonas sp. strains, showed sporadic sensitivity to the following antibiotics: ciprofloxacin $(\mathrm{n}=1)$, marbofloxacin $(\mathrm{n}=1)$ and tetracycline $(\mathrm{n}=1)$ (Figure 9). Pseudomonas 
express virulence factors and surface proteins affecting wound healing and the association with other pathogenic microorganisms increases resistance to antibiotics even more, making clinical management of infection a real challenge (Serra et al., 2015).

Among the myriad of microorganisms isolated from the bite wounds, a strain of Actinobacillus sp. was also found to have a very high sensitivity profile towards ampicillin, amoxicillin, clindamycin, enrofloxacin, marbofloxacin, ciprofloxacin and cephalexin (Figure 10).

Although Bacteroides species are anaerobic, they are transiently aero tolerant (Baughn and Malamy, 2004) and thus can survive in the bite wound. Bacteroides are resistant to a wide variety of $\beta$-lactams antibiotics, aminoglycosides, erythromycin and tetracycline (Salyers et al., 2004). Bacteroides strain isolated from a bitten wound was only sensitive to clindamycin and ciprofloxacin (Figure 11). Bacteroides species may become a reservoir for resistance in other, more highly pathogenic bacterial strains (Salyers et al., 2004).

Analyzing the sensitivity profile, it is found that isolated bacterial biotypes respond to most antibiotics tested, but in different ways. Following the percentage sensitivity ratio of the tested bacterial strains $(n=44)$, it was found that the most active antibiotics were: ciprofloxacin $(n=39)$,

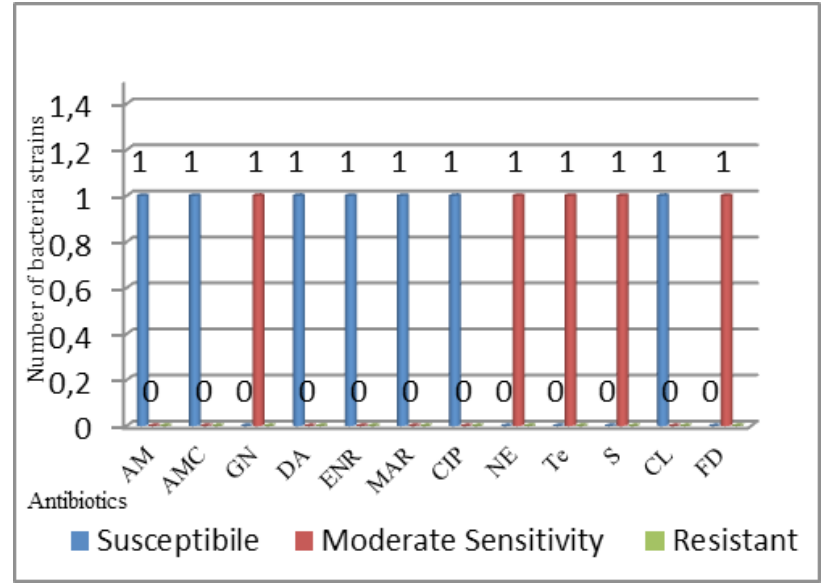

Figure 10. Antibiotic susceptibility profile of Actinobacillus sp.

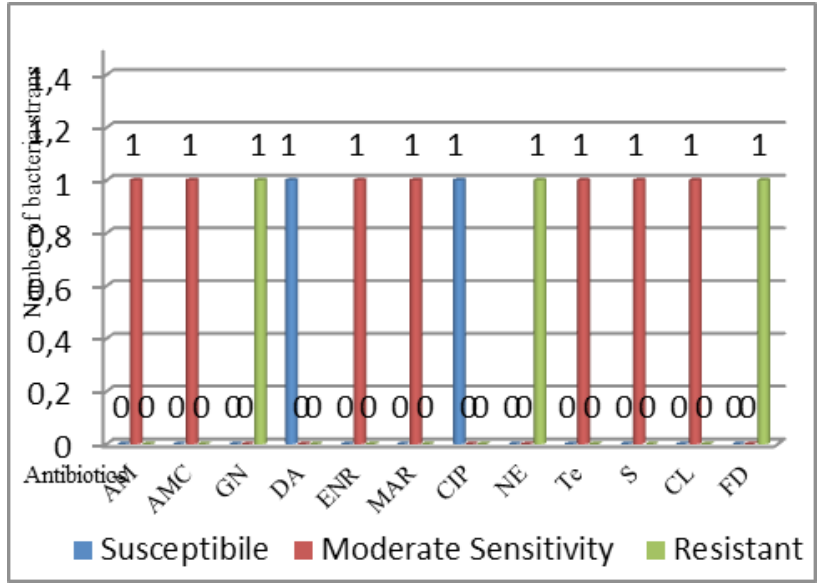

Figure 11. Antibiotic susceptibility profile of Bacteroides sp.

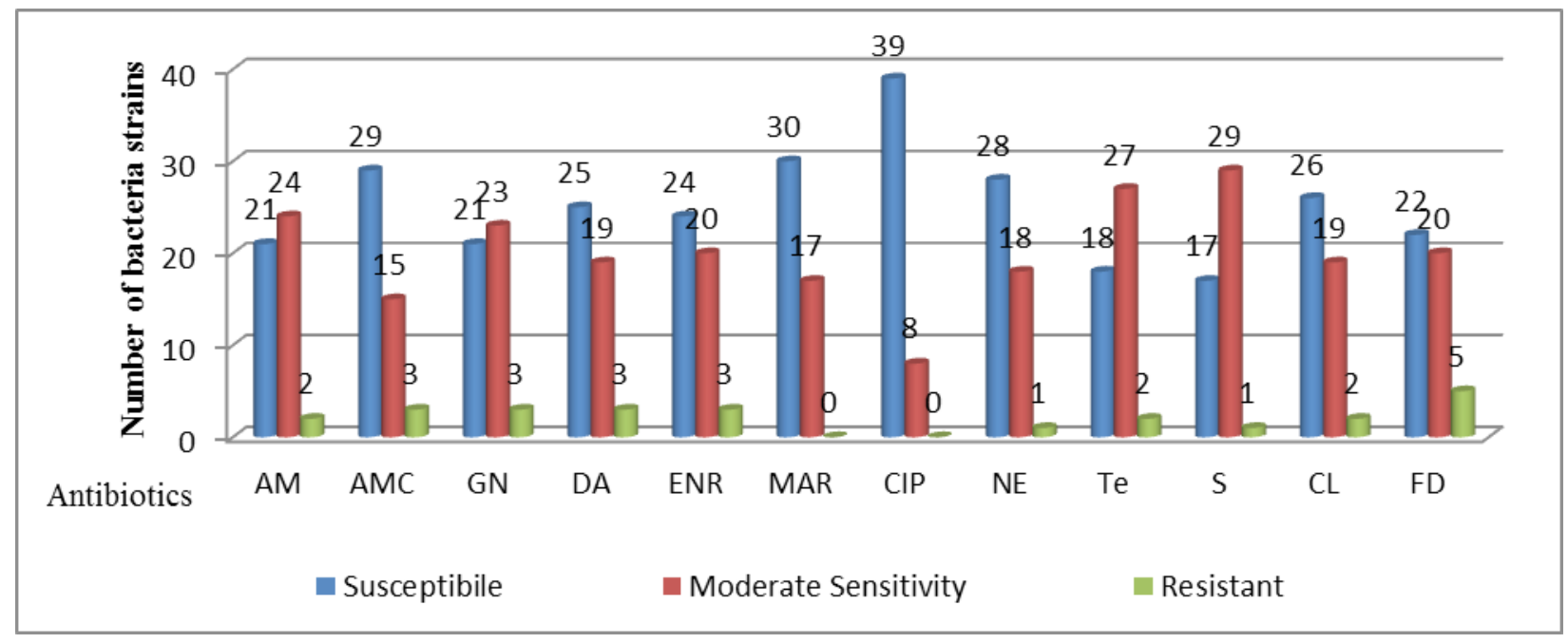

Figure 12. Variability of sensitivity and microbial resistance, of bacterial strains isolated from bite wounds 
marbofloxacin ( $\mathrm{n}=30)$, amoxicillin/clavulanic acid $(n=29)$, neomycin $(n=28)$, cephalexin $(n=26)$, clindamycin $(n=25)$, enrofloxacin $(n=24)$, fusidic acid $(n=22)$, gentamicin $(n=21)$, ampicillin $(n=21)$, tetracycline $(\mathrm{n}=18)$ streptomycin $(\mathrm{n}=17)$ (Figure 12.).

Antibiotic sensitivity results revealed a low microbial resistance to the panel of antibiotics tested $(\mathrm{n}=12)$. Bacterial biotypes were resistant to fusidic acid $(n=5)$, amoxicillin-clavulanic acid $(n=3)$, clindamycin $(n=3)$, enrofloxacin $(n=3)$, cephalexin $(\mathrm{n}=2)$, ampicillin $(\mathrm{n}=2)$, tetracycline $(n=2)$, gentamicin $(n=3)$, streptomycin $(n=1)$ and neomycin $(\mathrm{n}=1)$. Resistance to the fluoroinolones: marbofloxacin and ciprofloxacin was notidentified.

The bacterial strains in our study showed a very good sensitivity and a low resistance to the antibiotics commonly used in wound therapy. In this situation, we can argue that the proper application of prophylactic treatment with antibiotics, immediately after the trauma occurs, is of major importance in the clinical evolution.

However, the anti-infectious therapy must be supported by the antibiogram, because without targeted therapy, antibiotics stimulate the resistance mechanisms of the bacteria, which can lead to the emergence of antibiotic-resistant strains with well-known consequences (at present).

Therefore, as our study has shown, microorganisms involved in wound profusion are common, but they are recognized for their infectious capacity. Oral cavity microbiota and coetaneous microbiota represent important reservoirs of pathogenic or conditioned pathogenic commensal microorganisms.

For a better understanding of this risk, further studies with statistical relevance are needed, which will decrease the inherent biological variations, which may influence the microbiological results. Our approach was limited but the purpose was to highlight the importance of the bites produced by dogs and cats and to point out the infectious risk that veterinary medical personnel are subjected to during consultations.

\section{Conclusion}

The microbiota isolated from the wounds produced by the biteslargely reflects the microbiota of the oral cavity of the aggressor animals, to which are added the commensal microorganisms on the surface of the affected skin. Infected wounds, produced by the bite of dogs and cats in humans, present aerobic and anaerobic polymicrobial etiology, whose pathogenicity is reflected in the clinical evolution. Veterinary medical staff, like any patient, should approach the wounds created by the bite with the utmost seriousness, and resort to a specialized consultation and antiinfectious treatment prescribed on the basis of the antibiogram.

Acknowledgments. This research did not receive any specific grant from funding agencies in the public, commercial, or not-for-profit sectors.

\section{References:}

1. Abrahamian MF, Goldstein E (2011). Microbiology of Animal Bite Wound Infections, Clinical Microbiology Reviews.; 24(2): 231-246.

2. Alexander CJ, Citron DM, Hunt GS, Claros MC, Talan D, Goldstein EJ (1997). Characterization of saccharolyticBacteroides and Prevotella isolates from infected dog and cat bite wounds in humans, Journal of Clinical Microbiology. 35:406-411

3. Baughn A, Malamy M (2004). Molecular Basis for Aerotolerance of the Obligately Anaerobic Bacteroides Spp. In Nakano, Michiko; Zuber, Peter (eds.), Strict and Facultative Anaerobes: Medical and Environmental Aspects. CRC Press. p. 161. ISBN 978-1-904933-03-8

4. Carp Carare C, Guguianu E, Rimbu C (2015). Bacteriologie specială - Îndrumător de lucrări practice, Ed. USAMV Iasi, 2015, ISBN 978-973-147-180-8

5. DiNubile MJ, Lipsky BA (2004). Complicated infections of skin and skin structures: When the infection is more than skin deep, Journal of Antimicrobial Chemotherapy , 53(Suppl 2):ii37-50.

6. Brenner DJ, Hollis DG, Fanning GR, Weaver RE (1989). Capnocytophagacanimorsus sp. nov. (formerly CDC group DF-2), a cause of septicemia following dog bite, and $C$. cynodegmi sp. nov., a cause of localized wound infection following dog bite, Journal of Clinical. Microbiology. 27 : 231-235

7. Galperine T, Cazorla C, Blanchard E (2007). Streptococcus canis infections in humans: retrospective study of 54 patients, Journal of Infection; 55:23-26.

8. Gyles CL, Prescott JF, Songer JG, Thoen CO (2004). Pathogenesis of bacterial infections in animals, third edition, $\mathrm{Pb}$. Blackwell

9. Blackburn J, Tremblay E, Tsimiklis C, Thivierge C, Lavergne V (2013). Overwhelming sepsis after a cat bite, Canadian Journal of Infectious Diseases and Medical Microbiology; 24(2): e31-e32.

10. Maki DG, Agger WA (1988). Enterococcal bacteremia:clinical features, the risk of endocarditis, and management.Medicine (Baltimore) 67(4):248-269.

11. Maraki S, Kastanis G, Stafylaki D, Masunt S, Kapsetakis $P$, Scoulica E (2018). Pasteurellamultocida wound infection 
transmitted by a pet dog. GERMS ;8(4):214-217. doi: 10.18683/germs.2018.1149

12. Nielsen JP, Bisgaard M, Pedersen K B (1986). Production of toxin in strains previously classified as Pasteurella multocida. ActaPathologica Microbiology Immunology. Scandinavica B 94:203-204

13. Overall KL, Love M (2001). Dog bites to humansdemography, epidemiology, injury, and risk. Journal of the American Veterinary Medical Association.;218:1923-1934]

14. Peters V, Sottiaux M, Appelboom J, Kahn A (2004). Posttraumatic stress disorder after dog bites in children. The Journal of Pediatrics.;144:121-122.

15. Rao N, Jain S (1999). Pasteurella multocida meningitis from animal exposure. Infectious Diseases in. Clinical. Practice. 8:307-309

16. Rîmbu C (2010). Research regarding the implication of some bacterial species in dog and cat in mouth disease. Doctoral Thesis, USAMV Iasi

17. Rîmbu C, Guguianu E, Horhogea C, Carp-Carare M (2012) Stahylococcus aureus -Implication of the oral cavity disease at the dog and cat, Lucrări Ştiinţifice Vol. 55(3-4) MedicinăVeterinară, 2012, ISSN 1454-7406, pag 664-670

18. Salyers AA, Gupta A, Wang Y (2004). Human intestinal bacteria as reservoirs for antibiotic resistance genes. Trends in Microbiology. 12 (9): 412-6. doi:10.1016/j. tim.2004.07.004. PMID 15337162.

19. Serra R, Grande R, Butrico L, Rossi A, Settimio UF, Caroleo B, Amato B, Gallelli L, de Franciscis S. (2015). Chronic wound infections: the role of Pseudomonas aeruginosa and Staphylococcus aureus.Expert Rev Anti Infect Ther. 2015 May;13(5):605-13.

20. Talan D, M.D, Citron D, Abrahamian FM, Gregory JM, Goldstein E (1999). Bacteriologic Analysis of Infected Dog and Cat Bites, The New England Journal of Medicine; 340:85-92

21. Yaqub S, Bjørnholt JV, Enger AE (2004).Tularemia from a cat bite, Tidsskr Nor Laegeforen. ;124(24):3197-8.

22. Yuen JC, Malotky MV (2011). Francisella tularensis osteomyelitis of the hand following a cat bite: a case of clinical suspicion, Plastic and Reconstructive Surgery. 128(1):37e-9e. 